\title{
STATE OF THE ART AND EFFICIENCY OF ROBOTIC THERAPY IN UPPER LIMB REHABILITATION - A LITERATURE REVIEW
}

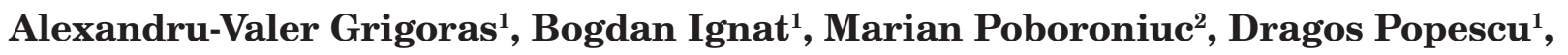 \\ Cristian Dinu Popescu ${ }^{1}$ \\ 1 "Gr. T. Popa" University of Medicine and Pharmacy, Iasi \\ 2 "Ghe. Asachi" Technical University, Iasi
}

\begin{abstract}
Stroke represents the main cause of disability in adults. The disability is caused by motor or sensitive impairment, loss of interjoint coordination, spasticity and pathological synergies that occur after stroke. The new directions in motor rehabilitation point at facilitating neuroplasticity by multimodal stimulation, like virtual reality, or by active assistance in task specific training, like rehabilitation robots associated or not with functional electrical stimulation (FES). The aim of this article is, on one hand, to analize the way in which the existing robots face the biomechanical chalenges of modeling the upper limb`s movements and, on the other hand, to evaluate the efficiency of robotics in rehabilitation, by pointing out the results of the existing clinical trials. Finally, possible directions for future research are discussed.
\end{abstract}

Keywords: rehabilitation robotics, exoskeleton, stroke, upper limb, rehabilitation

According to World Health Organisation, around 15 million people suffer a stroke anualy around the world, from which 5 million die and another 5 million remain with permanent disabilities. More than one quarter of stroke survivors become dependent in activities of daily living (ADL). It is estimated that aproximately $80 \%$ of survivors suffer from upper limb disability. Rehabilitation - hands-on therapy for several weeks - is attended in these pacients to treat hemiparesis and improve independence. It has been shown that therapist guided repetitive task specific training and constraint induced movement therapy (CIMT) have positive effects (1). However, even with intensive rehabilitation therapy from the acute phase, the probability of regaining the functionality of the afected limb remains low (2). A wide body of literature has promoted the idea that «repetitively trying to achieve a goal» is important for motor learning (3). In fact, repetitive goal-directed effort is so useful that even mental rehearsal of movement can improve arm motor impairment following stroke $(3,4)$. Last but not least, training with a physical therapist during a stay in a rehabilitation clinic is so costly that the average length of stay in rehabilitation units in the United States decreased from 31 to 14 days (5). Having this in mind, it is crucial that the pacient continues intensive therapy at home after hospital discharge.

With the intention of meeting these challenges, researchers began designing robotic devices for rehabilitation with the early 1990s. Robotic therapy represents a technique in which a robot participates alongside with the pacient, supervised or not by a therapist, in a process that is meant to facilitate and correct the rehabilitation phenomenon by enhancing neuroplasticity. Benefits that robotic therapy can bring are: providing intense repetitive training, giving quantitative feed-back, collecting real time data of motor performance, providing assistance or resistance similar to a hand-on technique and training in an engaging environment (6). 
The number of robotic devices and companies selling them grew constantly. An implicit idea was the partial automation; that is allowing patients to practice some of the repetitive aspects of rehabilitation therapy on their own, without the continuous presence of the rehabilitation therapist. However, there are little scientifically proven research directions in the field and the use of robotic devices worldwide is still rare.

The speculated benefic effects of robotic therapy are: neuroplasticity enhancement (since somatosensory input has been shown to drive cortical plasticity), reduced spasticity, and enhanced motivation (7). Motivation is a critical factor in rehabilitation and the best way to achieve it is to use an active assist mode in a virtual computer games-like environment (8).

The upper limb biomechanics consists of 7 degrees of freedom (DoF). One degree of freedom represents a point that can be moved along an axis. There are 3 DoFs in the shoulder, 2 in the elbow and 2 in the wrist. Of these, only 3 are necessary to move the hand in any point in space, but without the ability to grasp objects from any angle or direction. The central nervous system has the capacity to eliminate the redundancy and to find the best movement for a given task. This applies to global movements like walking, swimming, running or to fine movements like grasping or cathing an object. In the hemiparetic pacient, the interjoint coordination is severely altered and rehabilitation is probably linked to regaining of a coordinated movement pattern and to limiting of redundant movement (9). Also, in stroke pacients, additional movement apear, as are movements of the trunk (10). The speed of the movement is lower and the quality of movement is reduced by segmentation and lack of coordination (11).

Kinematic analysis of the time course of joint rotations showed a disruption of the relative timing between shoulder and elbow movements during reaching in stroke patients (12). Also, it showed that healthy subjects have a greater variability in movement, that prevents the motor system from becoming overly rigid and unable to adapt to complex constrains that may occur in various environments (13).

Early robotic therapy used end-effector robots, the so called "manipulandum", or operational devices, which can sustain and guide the upper limb by a single distal contact point. In the past years, research has shifted towards exoskeletons - rigid or semirigid structures with one or multiple joints, more degrees of freedom, that respect the anatomy of the limb. Their main role is to annulate gravity and guide movement. Exoskeletons can be equiped with rubber bands, springs or actuators. While upper-limb manipulanda and lower limb exoskeletons have been used in the clinical practice for several years (see the manipulandum by $\operatorname{InMotion}^{\odot}$ and the Lokomat $^{\circ}$ by Hocoma), upper-limb exoskeletons have only recently been developed (mid 2000s) and their effects have been little studied. The first commercially available upper-limb exoskeleton for rehabilitation was released at the end of 2011 (14).

Before designing an exoskeleton, one should answer the question: what should the robot do? Intuitively, the primary target for research teams in the field was to build robots that could actively assist movement. Therefore, a robotic system traditionally comprises 3 major components: a mechanical structure with degrees of freedom consistent with the tasks to be executed, joint-controlling actuators, either electric or pneumatic (for loads in the range of tens of Newtons), or hydraulic (for loads in the range of thousands of Newtons), a computer generating the signals that control the robot joints consistent with a priori information on the tasks to be executed and knowledge on actual and previous operative conditions and environment (15).

Interestingly, the field of robotics for neuro-rehabilitation has paralleled the development of industrial robots. The main issue is that, unlike most industrial robots, rehabilitation robots need to be designed for safe, stable, and compliant operation in close physical contact with humans. Rehabilitation robotics began by using robots to passively mobilize patient's limbs during the first stages of rehabilitation (passive mode) when the patient is unable to move alone. However, the effectiveness of such passive movements for stimulating motor recovery was limited (16). Another paradigm in robotic therapy is the so called "cooperative arm therapy". This usually means two things: stimulation of the three most important senses of the pacient (visual, auditory and haptic) and providing the minimum level of assistance necessary to perform a specific task. (17). Several research groups developed robotic therapy devices for the arm, but the first robotic devices to be clinically tested were MIT-Manus, MIME, and the ARM Guide, which all focused on providing active assist exercises for elbow flexion/extension and for limited shoulder movements. All exoskeletons that can actively assist movement require actuators. The most popular are the electrical actuators which are easy to control, available and low in cost. Their drawback is that they don 't have the best power to weight ratio. Alternatives are: pneumatic actuators, which are 
lightweight and fast but have complex control algorithms and hydraulic motors which have the best power to weight ratio but need an expensive instalation that includes a pump, hoses, valves and electric motor (18).

Robots can be classified upon the number of possible DoFs they provide. The more DoFs a device is capable, the more complex and expensive it is, without that being a guarantee for clinical efficacy. Most of the robots that were studied had small and functionally insignificant effects.

MIT-Manus (Massachusetts Institute of Technology) was developed in 1994. It is a operational device, which provides 2 translational DoFs for elbow and shoulder motion. The first MIT-Manus clinical trials have used unimanual pointing movements in the horizontal plane. The robot helped the patient to perform the movement to the target, following a normative trajectory (minimum jerk trajectory). Its main design feature was modularity, the capacity to adapt - there are multiple variants of the model, with various DoFs. Impedance control refers to the system (actuators, sensors and computer) that is used in order to impose a desired behavior during a specified type of interaction with a robot. It modulates the way the robot reacts to mechanical perturbation from the patient or clinician and ensures a gentle compliant behavior (19). This seems to be the best control approach of the robot.

MIT-Manus was the first robot that was tested in a clinical trial. In this trial, 20 stroke patients with similar characteristics were randomly distributed in a test group and in a control group. The patients who received robotic therapy had reduced shoulder and elbow motor impairment according to the Motor Status Score in comparison with the control group. The groups still statistically significantly differed in motor impairment at a 3-year follow-up (20).

One engineering challenge is achieving the dual goal of both enough force production to move a pacient's limb and of backdrivability. Backdrivability means that the robot "gets out of the way" during voluntary movement, so that the patient experiences a device that moves easily even with weak forces. It is essential in keeping the patient engaged in the task and providing meaningful feedback about the succesful completion of a task.

Existing controllers for exoskeletons are mostly assistive. According to the definition in MarchalCrespo and Reinkensmeyer (21), this involves replicating the therapist's behavior to assist the impaired subject to accomplish specific tasks. This paradigm can be implemented using different control techniques. The simplest way to control any robotic device is to create a feedback loop: this closed-loop structure usually regulates the position or the interaction forces along a known reference (for example, a trajectory or a force field model) and can be applied either at the joint (exoskeleton) or at the end-effector level (manipulandum).

«Assistance when needed» is the best control strategy in recovery. It supplements movement but it doesn't take over if subject's movements are sufficient. Technical implementation of this strategy often consists of controlling the interaction forces between the robot and the patient. Generally, these control strategies use a healthy control spatial path to define the desired motion, in combination with a "virtual wall"/force field that determines the amount of supportive force when the individual deviates from the template (impedance control). At the initial stages of recovery, the robot can take charge (high impedance), whereas at the concluding stages of recovery, the user must contribute more to the prescribed motion (low impedance) (22).

As an end manipulator, The Mirror Image Movement Enabler (MIME) is similar in concept with the MIT-Manus, but it is able to move the forearm through a large range of positions and orientations. MIME was developed in 1998, and it consists of a system with 6 DoFs. The 6 DoFs of the robot allow the forearm to be positioned within a large range of positions and orientations in three-dimensional space. MIME has several modes: passive mode, in which the pacient is relaxed and the robot moves the limb towards a target with a predetermined trajectory; active-assistive mode, where the pacient initiates the movement and then works with the robot along the desired movement; active-constrained mode, in which the robot provides resistance in the direction of the desired movement and spring-like restoring forces perpendicular to the movement direction and bimanual mode, in which the subject attempts bilateral mirror image movements while the robot assists the affected limb by continuously moving the affected forearm to the contralateral forearm's mirror image position and orientation with the help of a position digitizer. The clinical trial involving MIME included 27 stroke patients treated for 2 months. The robot group had a statistically significant larger increase in the Fugl-Meyer score than the control group. The robot group also improved more strength and reach extent. At the 6-month follow-up, there were no differences in Fugl-Meyer score; however, the robot group improved more in the self-care and transfers sections of the Functional Independence Measure (23). 
The ARM Guide is a robot consisting of a linear slide for the forearm, with 6 DoFs and force sensing. It is simple and relatively inexpensive. The assisted rehabilitation measurement and guide requires that the patient moves the end of the robotic arm. The position and speed of movement of the robotic arm are represented by a cursor on a video screen, and the reaching movements are executed in one direction at a time. If the patient cannot move the robotic arm, the device will assist. A clinical trial was conducted on chronic stroke patients to assess the ARM Guide. At the end of the study, both groups had significantly improved supported reaching speeds, but improvements did not significantly differ between the two groups (24).

The first rehabilitation robot to be commercially available was the ARMin exoskeleton. The device allows precise 3D arm movement. The robot is fixet at a wall and the pacient sits beneath, in a wheelchair with the torso fixed. ARMin has a shell-like exoskeleton structure with 4 active and 2 passive DoFs which can flex/extend the elbow and make spatial shoulder movements. The device has a complex sensor and control hardware. Movement of arm is accomplished in two steps: first, the therapist moves the arm together with the robot following the desired trajectory. The robot records the movement pattern and then it assists repetitive movements on the recorded trajectory. ARMin uses the game supported therapy paradigm which consists of a virtual arm placed in a virtual environment (25). A study on chronic stroke patients showed significant improvements in 3 out of 4 of the patients after training with the newer version of the device (ARMin II) for 8 weeks. The gains in Fugl-Meyer score were very heterogenic, between 29 points and 3 points, sensory impairment seemingly playing an important role in lack of response to therapy. The patients also reported improvements in concentration, neglect, well-being and posture (26).

There are a number of commercially available rehabilitation devices for the upper limb. Among the most sophisticated are the 7 DoFs ArmeoPower active exoskeleton, ArmeoSpring passive exoskeleton and ArmeoBoom sling suspension system. Other examples of commercial devices include the mPower arm brace (Myomo Inc., Cambridge, MA), a 1 DoF portable arm brace which uses electromyogram (EMG) signals measured from the biceps and triceps muscles to generate assistive torques for elbow flexion/extension, and the Hand Mentor (Kinetic Muscles Inc., Tempe AZ), a 1 DoF wearable device for the rehabilitation of the wrist and fingers which provides force, position and EMG feedback and is actuated by an air muscle. The Robot Suit HAL-5 (CYBERDYNE Inc., Japan) is a full body exoskeleton for the disabled.

Another interesting project combining the active assisted mode of an actuated exoskeleton with the lightness of a passive orthosis is the LIMPACT exoskeleton. It is a hydraulically powered upper limb aluminium exoskeleton, equiped with four hydraulic rotational motors (18).

In a study that compared the effects of robotic versus conventional rehabilitation therapy conducted on 143 subjects, the Fugl Meyer assessments showed greater improvements in the robotic therapy group, but the absolute difference between groups was of weak significance (27).

Moreover, a metaanalysis of robot rehabilitation studies, involving both subacute and cronic patients, showed no significant overall effect in favor of robot-assisted therapy but significant improvement of upper limb function for upper arm robots. Nevertheless there was no significant impact on ADL (28).

One important issue that has been outlined is that the amount of improvement depends heavily on the severity of the motor deficit and the appropriate selection of patients. The most favorable candidates for robotic therapy are patients with some recovery of voluntary wrist and finger extension in the first month post stroke or patients in the middle range of the Fugl-Meyer arm score (29). Possible ways to get closer to the final goal of functional independent movement could be an early intervention, increased dosage and association with other neuroregeneration approaches.

An alternate approach to bulky, complex, motorized robots, are simpler, passive, orthotics-like robots. These devices are less costly, they enhance safety and they are more suited for home use, but provide fewer DoFs, no feedback, no adapted support adjustment. The T-WREX (Therapy Wihnington Robotic Exoskeleton) is such a device - an exoskeleton structure with elastic bands placed on it that allows 5 DoFs and a broad 3D working space. The number of elastic bands provides adapted levels of arm support. Additionally, it can be used as an input device for computer games with auditory and visual feedback. Assessment of movement is made using joint position sensors and a handgrip sensor. In a randomised controlled trial involving chronic stroke patients with moderate functional loss, training with the T-WREX was compared to conventional therapist-guided training (table-top training). The study showed significant improvements in arm movement ability in both groups, but all subjects preferred the T-WREX on the satisfac- 
tion survey questionnaires. The improvements obtained on the Fugl-Meyer score were similar to those obtained with actuated and more expensive robots like MIT-Manus or MIME (6). Pneu WREX is the pneumatically actuated version of the T-WREX and it was studied on chronic stroke patients. Robotic therapy patients showed a small supplementary motor improvement on the Fugl Meyer scale compared with conventional therapy patients (30).

Another direction of research is the design of lightweight hybrid FES-exoskeleton systems. Functional electrical stimulation (FES) therapy combines preprogrammed coordinated electrical muscular stimulation and manual assisted passive motion by a therapist to establish physiologically correct movement. In a randomized controlled study it has been shown that FES therapy had positive effects on upper-limb function, especially in patients with sub acute stroke. Patients that underwent FES therapy plus conventional therapy for 12 weeks improved significantly more than patients who only had conventional therapy (a difference of 24 points on the Fugl Meyer score - with 10 points showing clinically relevant change) $(31,32)$. Later studies propose to replace the therapist with a exoskeleton that would work in a parallel synchronized way to assist/complete the FES-based induced motion, FES generated contraction partially providing the necessary force during arm and forearm movement (33).

Our team is currently evaluating the efficacy of such a hybrid FES-exoskeleton system. The exoskeleton part consists of a left side medium size leather glove with metal "tendons" attached to it on the dorsal side of the hand for active finger flexionextension movements. The FES part consists of a two chanel electrical stimulator, one for interosseous muscles and the other for extensor digitorum muscle. Input information comes from a second leather glove with bending sensors attached, for the right hand. The system uses a software which allows the setting of electrical stimulation intensity and the posibility to follow the pacient's performance: number of movements, amplitude of movement, intensity of stimulation.

An even more innovative system is the myocontrolled neuroprosthesis integrated with a passive exoskeleton. According to the principle that the therapeutic effect of any intervention is augmented when it is applied simultaneously with the voluntary drive, the timing and intensity of the electrical muscle stimulation was controlled using EMG signals extracted from partially paralyzed muscles (34).

An interesting question is whether motivating software, aiming to promote normalized movement patterns and target specific areas of upper extremity weakness would become a mandatory component of any rehabilitative approach. Since progress depends on training intensity and training duration, the motivation of the patient turns out to be a key factor for an efficient rehabilitation (24).

Moreover, future research on the effects of robot-assisted therapy should separately address upper and lower arm training and concentrate on kinematical analysis to differentiate between genuine upper limb motor recovery and functional recovery due to compensation strategies by proximal control of the trunk and upper limb (27). To avoid compensatory strategies, the use of a multi-contact robotic device for the rehabilitation of coordination seems promising. However, the development of exoskeletons for rehabilitation is only beginning and numerous technological, physiological, and clinical challenges lie ahead.
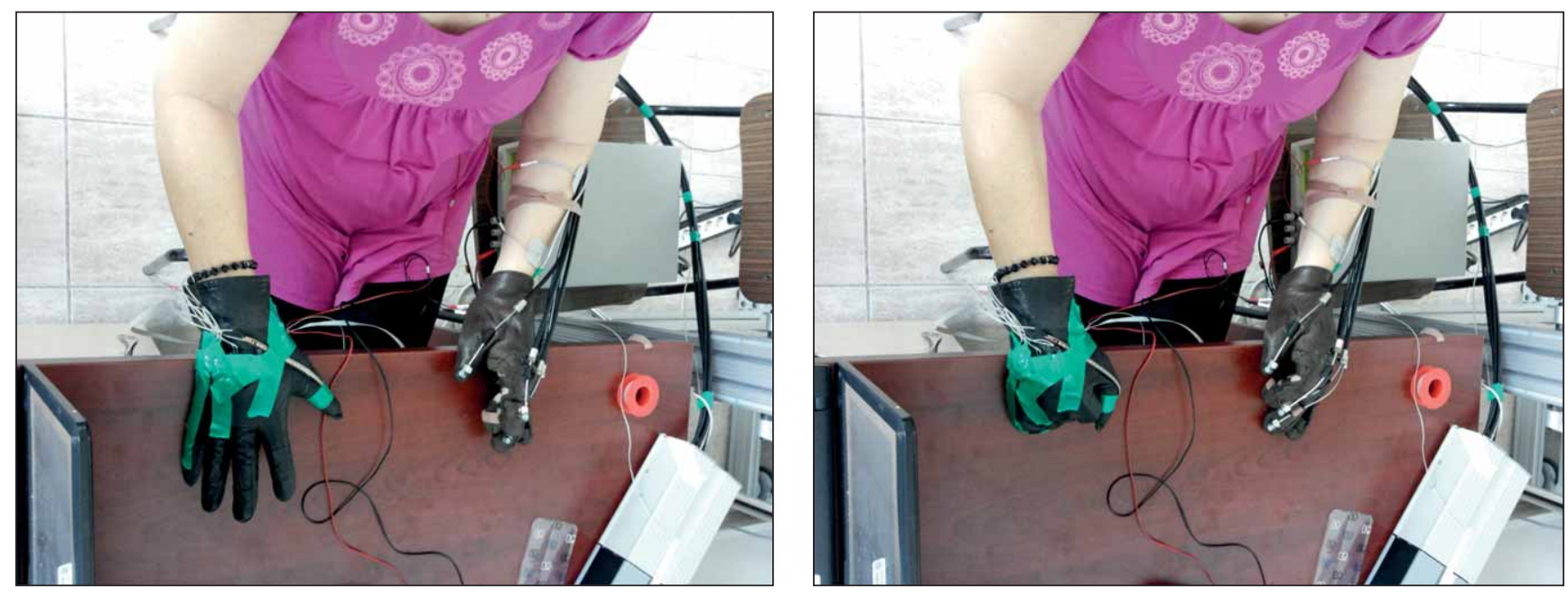

FIGURE 1. Hybrid FES-exoskeleton system for left hand rehabilitation: finger extension (left), finger flexion (right) 
It is clear that robotic therapy has a potential to reduce the burden on the healthcare system (at least in terms of human resources) and the most convenient picture is that of a robotic therapy room with several patients undergoing motivating software connected robotic training, all being assisted by a single therapist who would ensure that movements are correctly done. Another convenient picture is robotic training at home with remote performance assessment.

Nevertheless, one important fact is that the therapeutic benefits of robotic therapy are modest, and have not been shown to be decisively better than other less expensive approaches that can partially automate therapy and maybe offer the same amount of patient motivation. In other words, the necessity question remains unanswered. There is little moti-

\section{REFERENCES}

1. Kwakkel G., Veerbeek J., van Wegen E. et al. Constrained induced movement therapy after stroke, Lancet Neurology, 2015; 14: 224-234

2. Rosamond W., Flegal K., Friday G., et al. Heart disease and stroke statistics-2007 update: A report from the American Heart Association Statistics Committee and Stroke Statistics Subcommittee, Circulation, 2007; 115: 69-171

3. O'Sullivan S., Schmitz T. Physical rehabilitation, 5th edition, F.A. Davis Company Philadelphia, 2007

4. Levin M.F., Weiss P.L., Keshner E.A. Emergence of virtual reality as a tool for upper limb rehabilitation: incorporation of motor control and motor learning principles, Phys Ther, 2015; 95: 415-425

5. Schmidt S.M., Guo L., Scheer S.J. Changes in the status of hospitalized stroke patients since inception of the prospective payment system in 1983, Arch Phys Med. Rehabil, 2002; 83: 894-898

6. Housman S., Rahman T., Sanchez R. et al. Arm-Training with T-WREX After Chronic Stroke: Preliminary Results of a Randomized Controlled Trial, Proceedings of the 2007 IEEE $10^{\text {th }}$ International Conference on Rehabilitation Robotics

7. Merzenich M.M., Jenkins W.M. Reorganization of cortical representations of the hand following alterations of skin inputs induced by nerve injury, skin island transfers, and experience, Journal of Hand Therapy, 1993; 6: 89-104

8. Reinkensmeyer D.J., Housman S.J. If I can't do it once, why do it a hundred times?: Connecting volition to movement success in a virtual environment motivates people to exercise the arm after stroke, In: Virtual Rehabilitation, Elsevier, 2007: 44-48

9. Crocher V., Sahbani A., Robertson J. et al. Constraining Upper Limb Synergies of Hemiparetic Patients Using a Robotic Exoskeleton in the Perspective of Neuro-Rehabilitation, IEEE Transactions on Neural Systems and Rehabilitation Engineering, 2012; 20: 247-257

10. Michaelsen S.M., Dannenbaum R., Levin M.F. Task-specific training with trunk restraint on arm recovery in stroke: randomized control trial, Stroke, 2006; 37: 186-192

11. Cirstea M.C., Mitnitski A.B., Feldman A.G. et al. Interjoint coordination dynamics during reaching in stroke, Exp Brain Res, 2003; 151:289-300

12. Kordelaar van J., Wegen van E.E., Kwakkel G. Unraveling the interaction between pathological upper limb synergies and compensatory trunk movements during reach-to-grasp after stroke: a cross-sectional study. Exp.Brain Res., 2012; 221: 251-262 vation for most clinics to buy expensive robots until it is proven that they yield therapeutic or cost benefits that are substantially better than current approaches. This field will most likely have to evolve to place more focus on mechanisms of motor plasticity, allowing an optimization of the technological approach, and eventually improve the benefits of robotic therapy. It remains to be established whether the fundamental limits of plasticity would allow an expensive robot to be cost efficient.

\section{Acknowledgements}

This paper has been supported within the frame of "An Intelligent haptic robot glove for the pacients suffering a cerebrovascular accident" (IHRG) project, under the contract number 150/2012.

13. Patterson T.S., Bishop M., McGuirk T., et al. Reliability of Upper Extremity Kinematics While Performing Different Tasks in Individuals With Stroke, Journal of Motor Behavior, 2011; 43: 121-130

14. Riener R., Guidali M., Keller U., et al. Transferring Armin to the clinics and industry. Top. Spinal Cord Inj. Rehabil. 2011; 17: 54-59

15. Pignolo L. Robotics in Neuro-Rehabilitation, Journal of Rehabilitation Medicine, 2009; 41: 955-960

16. Lynch D., Ferraro M., Krol J., et al. Continuous passive motion improves shoulder joint integrity following stroke, Clin. Rehabi, 2005; 19: 594-599

17. Patton J.L., Mussa-Ivaldi F.A. Robot assisted adaptative training: custom force fields for teaching movement patterns, IEEE Rev. Biomed. Eng., 2002; 51: 636-646

18. Otten A., Voort C., Stienen A. et al. LIMPACT: A Hydraulically Powered Self-Aligning Upper Limb Exoskeleton, ASME Transactions on mechatronics, 2015; 20: 2285-2298

19. Krebs H.I., Ferraro M., Buerger S.P., et al. Rehabilitation robotics: pilot trial of a spatial extension for MIT-Manus, J Neuroeng Rehabil, 2004; $1: 5-9$

20. Krebs H.I., Hogan N., Aisen M. Robot-Aided Neurorehabilitation, IEEE Trans Rehabil Eng. 1998; 6: 75-87

21. Marchal-Crespo L., Reikensmeyer D. Review of control strategies for robotic movement training after neurologic injury, J Neuroeng Rehabil., 2009; 6: 20-35

22. Fleerkotte B.M., Koopman B., Buurke J. et al. The effect of impedance-controlled robotic gait training on walking ability and quality in individuals with chronic incomplete spinal cord injury: an explorative study, J Neuroeng Rehabil. 2014; 11: 26-36

23. Lum P.S., Burgar C.G., Loos, van der, M. et al. MIME robotic device for upper-limb neurorehabilitation in subacute stroke subjects: A follow-up study, Journal of Rehabilitation Research and Development, 2006; 43: 631-642

24. Reinkensmeyer D.J., Kahn L., Averbuch M. et al. Understanding and treating arm movement impairment after chronic brain injury: Progress with the ARM Guide, Journal of rehabilitation research and development, 2000; 37: 653-662

25. Nef T., Mihelj M., Riener R. ARMin: a robot for patient-cooperative arm therapy, Medical \& Biological Engineering \& Computing, 2007; 45: 887-900

26. Staubli P., Nef T., Klamroth-Marganska V. et al. Effects of intensive arm training with the rehabilitation robot ARMin II in chronic stroke 
patients: four single-cases, Journal of NeuroEngineering and Rehabilitation, 2009; 6: 46-51

27. Klamroth-Marganska V., Blanco J., Campen K. et al. Three-dimensional, task-specific robot therapy of the arm after stroke: a multicentre, parallel-group randomised trial, Lancet Neurol., 2014; 13:159-166

28. Prange G., Michiel M., Jannink J. et al. Systematic review of the effect of robot-aided therapy on recovery of the hemiparetic arm after stroke, Journal of Rehabilitation Research \& Development, 2006; 43: 171-184

29. Kwakkel G., Kollen B., Krebs H. Effects of Robot-assisted therapy on upper limb recovery after stroke: A Systematic Review, Neurorehabil Neural Repair, 2008; 22: 111-121

30. Reinkensmeyer D., Wolbrecht E., Chan V. Comparison of 3D, Assist-as-Needed Robotic Arm/Hand Movement Training Provided with Pneu-WREX to Conventional Table Top Therapy Following Chronic Stroke, Am J Phys Med Rehabil, 2012: 232-241
31. Thrasher T.A., Zivanovic V., Mcllroy W. et al. Rehabilitation of reaching and grasping function in severe hemiplegic patients using functional electrical stimulation therapy, Neurorehabil Neural Repair. 2008; 22: 706-714

32. Kawashima N., Popovic M., Zivanovic V. Effect of Intensive Functional Electrical Stimulation Therapy on Upper-Limb Motor Recovery after Stroke: Case Study of a Patient with Chronic Stroke, Physiotherapy Canada, 2013; 65: 20-28

33. Serea F., Poboroniuc M., Hartopanu S. Preliminary tests on a hybrid upper arm exoskeleton for upper arm rehabilitation for disabled patients, 2014 International Conference and Exposition on Electrical and Power Engineering

34. Ambrosini E., Ferrante S., Schauer T. et al. A myocontrolled neuroprosthesis integrated with a passive exoskeleton to support upper limb activities, Journal of Electromyography and Kinesiology, 2014; 24: 307-317 